\title{
Determinants of activation for self-management in patients with COPD
}

\author{
This article was published in the following Dove Press journal: \\ International Journal of COPD \\ I August 2016 \\ Number of times this article has been viewed
}

\section{YJG Korpershoek ${ }^{1-3}$ \\ ID Bos-Touwen² \\ JM de Man-van Ginkel ${ }^{2,4}$ \\ J-WJ Lammers ${ }^{3}$ \\ MJ Schuurmans ${ }^{1,2}$ \\ JCA Trappenburg ${ }^{2}$}

'Research Group Chronic Illnesses, Faculty of Health Care, University of Applied Sciences Utrecht, ${ }^{2}$ Department of Rehabilitation, Nursing Science \& Sports, University Medical Center Utrecht, ${ }^{3}$ Department of Respiratory Medicine, Division of Heart \& Lungs, University Medical Center Utrecht, ${ }^{4}$ Nursing Science, Program in Clinical Health Science, University Medical Center Utrecht, Utrecht, the Netherlands

\section{Video abstract}

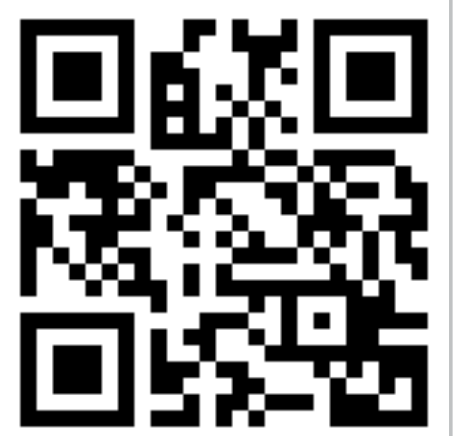

Point your SmartPhone at the code above. If you have a QR code reader the video abstract will appear. Or use:

http://youtu.be/0r798882F28

Correspondence: YJG Korpershoek Faculty of Health Care, University of Applied Sciences Utrecht, Heidelberglaan 7, 3584 CS, Utrecht, PO box | 20| | -350 |

AA Utrecht, the Netherlands

Tel +3I 638763949

Email yvonne.korpershoek@hu.nl
Background: COPD self-management is a complex behavior influenced by many factors. Despite scientific evidence that better disease outcomes can be achieved by enhancing selfmanagement, many COPD patients do not respond to self-management interventions. To move toward more effective self-management interventions, knowledge of characteristics associated with activation for self-management is needed. The purpose of this study was to identify key patient and disease characteristics of activation for self-management.

Methods: An explorative cross-sectional study was conducted in primary and secondary care in patients with COPD. Data were collected through questionnaires and chart reviews. The main outcome was activation for self-management, measured with the 13-item Patient Activation Measure (PAM). Independent variables were sociodemographic variables, selfreported health status, depression, anxiety, illness perception, social support, disease severity, and comorbidities.

Results: A total of 290 participants (age: $67.2 \pm 10.3$; forced expiratory volume in 1 second predicted: 63.6 \pm 19.2 ) were eligible for analysis. While poor activation for self-management (PAM-1) was observed in $23 \%$ of the participants, only $15 \%$ was activated for self-management (PAM-4). Multiple linear regression analysis revealed six explanatory determinants of activation for selfmanagement $(P<0.2)$ : anxiety ( $\beta$ : $-0.35 ;-0.6$ to -0.1$)$, illness perception $(\beta:-0.2 ;-0.3$ to -0.1$)$, body mass index (BMI) ( $\beta$ : $-0.4 ;-0.7$ to -0.2$)$, age $(\beta:-0.1 ;-0.3$ to -0.01$)$, Global Initiative for Chronic Obstructive Lung Disease stage ( 2 vs $1 \beta$ : $-3.2 ;-5.8$ to $-0.5 ; 3$ vs $1 \beta:-3.4 ;-7.1$ to 0.3 ), and comorbidities ( $\beta$ : $0.8 ;-0.2$ to 1.8 ), explaining $17 \%$ of the variance.

Conclusion: This study showed that only a minority of COPD patients is activated for selfmanagement. Although only a limited part of the variance could be explained, anxiety, illness perception, BMI, age, disease severity, and comorbidities were identified as key determinants of activation for self-management. This knowledge enables health care professionals to identify patients at risk of inadequate self-management, which is essential to move toward targeting and tailoring of self-management interventions. Future studies are needed to understand the complex causal mechanisms toward change in self-management.

Keywords: COPD, self-management, patient activation, patient and disease characteristics

\section{Introduction}

COPD is one of the most prevalent chronic diseases worldwide and the fourth leading cause of mortality. ${ }^{1,2}$ Increased burden of COPD is expected due to aging of the population and continued exposure to COPD risk factors. ${ }^{1,3}$ To address the burden on both patients and society, self-management has become increasingly important. ${ }^{4-6}$ Selfmanagement is defined as “an individual's ability to detect and manage symptoms, treatment, physical and psychosocial consequences, and lifestyle changes inherent in living with a chronic condition". ${ }^{7}$ Self-management can support COPD patients to 
manage their symptoms, prevent complications, and make adequate decisions on medication, exercise, breathing techniques, diet, and contacting health care providers. ${ }^{4,8,9}$

The pivotal objective of self-management interventions is to change health behaviors and to equip patients with skills to actively participate in the management of their disease. ${ }^{4,10}$ Previous research has shown that self-management interventions have positive effects on disease outcomes, healthrelated quality of life, and health care costs. ${ }^{5,11,12}$ A substantial proportion of COPD patients, however, do not respond or comply with self-management interventions. ${ }^{5,10,13}$ The large variance in effectiveness between patients presumes that it is unlikely that one intervention fits all patients. ${ }^{10,13}$ Health care professionals play a major role in providing self-management support, but patients' initial self-management capabilities are often not determined by these professionals, frequently resulting in a "one size fit all approach". ${ }^{10,14}$

To identify COPD patients who are more engaged in self-management and patients who encounter difficulties in performing adequate self-management, more insight into patient and disease characteristics associated with self-management behavior is needed. ${ }^{8,10}$ The process toward adequate self-management requires an increase in knowledge, skills, and confidence for self-management, which is defined as the level of activation for self-management. ${ }^{14}$ Higher levels of activation reflect better capacity to self-manage one's disease. ${ }^{14,15}$ In a recent study, we investigated factors associated with activation for self-management in a large population of patients with various chronic diseases (eg, diabetes mellitus type II, chronic heart failure, chronic renal disease, and COPD). ${ }^{16}$ This study identified age, body mass index (BMI), educational level, financial distress, physical health status, depression, illness perception, social support, and underlying disease as important determinants, explaining 16\% of variance in activation for self-management. ${ }^{16}$ These associations were disease transcending except for social support. More specific, the association between COPD and activation was dependent on social support, while this was not observed for other conditions. ${ }^{16}$ In this study, no specific COPD-related factors were taken into account and, therefore, factors explaining variance in activation for self-management in COPD patients specifically remain unclear. Previous studies have shown that COPD-specific characteristics such as dyspnea and disease severity may also be related to self-management behavior. ${ }^{8,17,18}$ Investigating the association between COPDspecific determinants and activation for self-management, combined with previously investigated determinants, may contribute to a thorough understanding of factors influencing self-management behavior in COPD patients.
To move toward the development of targeted and tailored self-management interventions with improved efficiency and (cost-) effectiveness, knowledge on key patient and disease characteristics of activation for self-management in COPD patients specifically is needed. Therefore, the objective of this study was to identify key determinants of activation for self-management in patients with COPD.

\section{Methods}

\section{Study design}

A descriptive study was performed with a cross-sectional research design. The study was conducted in one secondary and two primary care settings in the Netherlands and was part of a larger study. ${ }^{16}$ The study was approved by the Medical Research Ethics Committee of the University Medical Center of Utrecht.

\section{Study population and recruitment}

Patients diagnosed with mild-to-very severe COPD were selected by the attending physician according to the following inclusion criteria: a clinical diagnosis of COPD meaning a post-bronchodilator forced expiratory volume in 1 second $\left(\mathrm{FEV}_{1}\right)$ /forced vital capacity $(\mathrm{FVC})$ ratio $<70 \%$ and age above 40 years. In secondary care, patients should have visited the outpatient clinic in the past 6 months to reduce the risk of including patients who are deceased or are no longer under treatment. Exclusion criteria were a diagnosis of lung cancer, cognitive impairments, language or communication problems, and a life expectancy of less than 3 months.

The sample size was calculated to allow sufficient power for a multiple linear regression analysis using 20 variables. According to the ratio of number of predictor variables to number of participants (1:10), a sample size of at least 200 participants was required. ${ }^{19}$ Patients were selected by chart review according to the inclusion and exclusion criteria. Patients received an invitation letter from their attending physician to participate in this study. Attached with the invitation letter, patients also received a letter with study information, an informed consent form, a questionnaire, and a pre-addressed return envelope. To enhance recruitment rates, patients were sent a reminder after 3 weeks if the IC form was not returned. By signing the IC form, patients gave consent to consult their medical chart to obtain additional information.

\section{Data collection}

Data were collected by means of administering a questionnaire and medical chart review. The questionnaire was a composition of Dutch-validated questionnaires and a set of questions to determine sociodemographic characteristics. 
The primary outcome activation for self-management was measured by the Patient Activation Measure (PAM-13), a 13-item instrument that assesses self-reported knowledge, skills, and confidence for self-management. ${ }^{14,20,21}$ A positive change in activation has shown to be associated with positive changes in various self-management behaviors. ${ }^{15}$ Items are scored on a five-point scale. The sum of these scores is converted in a $0-100$ point scale. ${ }^{20,22}$ Based on cut-off points for the four levels of activation - level 1 ( $\leq 47.0$ points), level 2 (47.1-55.1 points), level 3 (55.2-67 points), and level 4 ( $\geq 67.1$ points) - the individual patients' level of activation can be determined. ${ }^{20,22} \mathrm{~A}$ higher level refers to higher activations scores. ${ }^{14}$ Patients in level 1 are often passive and lack confidence for self-management resulting in low self-management engagement. Patients in level 2 become aware that they should be involved in their care, although there remain gaps in knowledge and skills. Patients in level 3 gain confidence for self-management and start to take action. The fourth, and highest, level of activation includes patients who have adopted new behaviors and are challenged to maintain these behaviors over time. Therefore, patients with higher levels of activation are considered to be better self-managers. ${ }^{14,15,23}$

The PAM-13 is translated in Dutch and validated in COPD patients showing good internal consistency $(\alpha=0.88)$. Item-rest correlations were moderate-to-strong and test-retest reliability was moderate. ${ }^{21}$

Determinants of activation for self-management were measured using the following instruments. Health status was measured by the Short Form-12 Health Survey (SF-12), a short version of the Short Form-36 (SF-36). ${ }^{24,25}$ The 12-item SF-12 measures both physical and mental health. ${ }^{26}$ Item scores result in two summary scores on a 0-100 point scale. Higher scores refer to a better health status. Presence of anxiety or depression was measured by the Dutch-validated Hospital Anxiety and Depression Scale (HADS). ${ }^{27,28}$ The HADS includes two seven-item subscales (anxiety and depression) both with a score range of $0-21 .{ }^{28}$ Higher scores refer to a higher state of anxiety or depression, with cut-off point $\geq 11$ indicating a depression or anxiety disorder. The Brief Illness Perception Questionnaire (B-IPQ) was used to measure illness perception. ${ }^{29}$ The B-IPQ consists of eight items, each scored on a scale from 1 to 10 , resulting in an overall score (range: 0-80). Higher scores indicate a more negative illness perception. Assessment of reproducibility was performed with Dutch COPD patients and showed moderate to good reliability. ${ }^{29}$ The 12 -item Multidimensional Scale of Perceived Social Support was used to assess social support. ${ }^{30,31}$ Items were scored on a seven-point scale. Higher scores indicate higher perceived support. ${ }^{30,31}$ Validity and reliability were confirmed by Dutch cardiac patients and their partners. ${ }^{32}$

Sociodemographic characteristics included age, sex, BMI, ethnicity, socioeconomic status, living situation, and smoking habits. Socioeconomic status was operationalized in three separate variables: educational level, financial distress, and care allowance as a proxy for income. Operationalization of these determinants is detailed in Table 1.

Disease characteristics included COPD severity, COPD duration, current exacerbation, and comorbidities. Severity of COPD was obtained from the medical chart and classified into four stages of Global Initiative for Chronic Obstructive Lung Disease (GOLD), which were determined using $\mathrm{FEV}_{1} / \mathrm{FVC}$ and $\mathrm{FEV}_{1} \%$ predicted data. ${ }^{1}$ In case of missing lung function data, GOLD stage as reported by the physician was used. To complement $\mathrm{FEV}_{1} \%$ predicted in the classification of $\mathrm{COPD}$ severity, dyspnea was measured by the five-point Medical Research Council (MRC) scale. A higher score indicates a higher degree of perceived breathlessness. ${ }^{33} \mathrm{COPD}$ duration was determined by number of years since diagnosis. Current exacerbation at the time of the measurement was examined by asking whether patients currently used a course of antibiotics and/or prednisolone. Furthermore, comorbidities obtained from chart review were assessed by the Charlson comorbidity index (CCI) ${ }^{34,35}$ The CCI is based on ICD-10 (International Classification of Diseases - tenth revision) codes and defines 19 comorbidities. A weighted score, based on the relative risk of mortality at 1 year, was assigned to each comorbidity with a total range of $0-37 .^{35}$

\section{Statistical analysis}

Statistical analysis was performed using SPSS 21.0 (IBM Corporation, Armonk, NY, USA). ${ }^{36}$ Descriptive statistics were used to describe baseline characteristics. Means and standard deviations were used to describe continuous variables, whereas frequencies and percentages were used for categorical variables.

Patients were excluded when all 13 questions of the PAM-13 were answered identically or showed more than seven missing. ${ }^{21,22}$ Analysis of missing values of all determinants was performed and showed $2 \%$ missing variables, distributed among $31 \%$ of the cases. Multiple imputation was used to deal with missing data, since this may reduce bias when data are missing at random. ${ }^{37}$ Data analysis was performed in ten imputed data sets.

Univariate linear regression analysis was used to analyze the association between single determinants and activation for self-management, rather as a method for selecting 
Table I Baseline characteristics of the study population

\begin{tabular}{|c|c|}
\hline Sociodemographic characteristics & Total $(n=290)$ \\
\hline \multicolumn{2}{|l|}{ Sex } \\
\hline Male & $184(63.4 \%)$ \\
\hline Female & $105(36.2 \%)$ \\
\hline Age, years (mean $\pm S D)$ & $67.2 \pm 10.3$ \\
\hline $\mathrm{BMI}($ mean $\pm \mathrm{SD})$ & $26.6 \pm 4.7$ \\
\hline \multicolumn{2}{|l|}{ Ethnicity } \\
\hline Dutch & $268(92.4 \%)$ \\
\hline Other & $19(6.6 \%)$ \\
\hline \multicolumn{2}{|l|}{ Marital status ${ }^{\mathrm{a}}$} \\
\hline Married & $192(66.2 \%)$ \\
\hline Not married & $98(33.8 \%)$ \\
\hline \multicolumn{2}{|l|}{ Living situation } \\
\hline Living alone & $72(24.8 \%)$ \\
\hline Not living alone & $213(73.4 \%)$ \\
\hline \multicolumn{2}{|l|}{ Education level ${ }^{b}$} \\
\hline Low & $126(43.4 \%)$ \\
\hline Medium & III (38.3\%) \\
\hline High & $46(15.9 \%)$ \\
\hline \multicolumn{2}{|l|}{ Current working } \\
\hline Yes & $48(16.6 \%)$ \\
\hline No & $237(81.7 \%)$ \\
\hline \multicolumn{2}{|l|}{ Financial distress } \\
\hline None & $|2|(4 \mid .7 \%)$ \\
\hline Low & $129(44.5 \%)$ \\
\hline High & $33(11.4 \%)$ \\
\hline \multicolumn{2}{|l|}{ Care allowance ${ }^{c}$} \\
\hline Received & $119(41.0 \%)$ \\
\hline Not received & $159(54.8 \%)$ \\
\hline \multicolumn{2}{|l|}{ Smoking } \\
\hline Never & $25(8.6 \%)$ \\
\hline Former & $173(59.7 \%)$ \\
\hline Current & $89(30.7 \%)$ \\
\hline Average smoking (pack-years) (mean $\pm \mathrm{SD}$ ) & $36.6 \pm 24.6$ \\
\hline Social support (MSPSS) (mean \pm SD) & $60.6 \pm 17.4$ \\
\hline \multicolumn{2}{|l|}{ HADS } \\
\hline Depression (mean \pm SD) & $5.6 \pm 4.1$ \\
\hline Anxiety (mean \pm SD) & $5.7 \pm 4.3$ \\
\hline \multicolumn{2}{|l|}{ Health status (SF-I2) } \\
\hline Physical component (mean \pm SD) & $45.6 \pm 24.5$ \\
\hline Mental component (mean \pm SD) & $61.7 \pm 23.4$ \\
\hline Illness perception (B-IPQ) (mean \pm SD) & $40.1 \pm 12.0$ \\
\hline Activation (PAM) (mean $\pm S D)$ & $54.7 \pm 10.4$ \\
\hline \multicolumn{2}{|l|}{ Illness duration } \\
\hline$\leq 2$ years & $42(14.5 \%)$ \\
\hline $2-5$ years & $69(23.8 \%)$ \\
\hline$>5$ years & $135(46.6 \%)$ \\
\hline $\mathrm{FEV}, \%$ predicted $($ mean $\pm \mathrm{SD})$ & $63.6 \pm 19.2$ \\
\hline \multicolumn{2}{|l|}{ GOLD stage $^{d}$} \\
\hline I (mild) & $93(32.1 \%)$ \\
\hline II (moderate) & $133(45.9 \%)$ \\
\hline III (severe) & $40(13.8 \%)$ \\
\hline IV (very severe) & $14(4.8 \%)$ \\
\hline \multicolumn{2}{|l|}{ MRC score } \\
\hline$\leq 3$ & $183(63.1 \%)$ \\
\hline$>3$ & $105(36.2 \%)$ \\
\hline Charlson comorbidity index $(\text { mean } \pm S D)^{e}$ & $2.5 \pm 1.5$ \\
\hline
\end{tabular}

(Continued)
Table I (Continued)

\begin{tabular}{ll}
\hline Sociodemographic characteristics & Total $(\mathbf{n}=\mathbf{2 9 0})$ \\
\hline Current exacerbation & \\
No & $224(77.2 \%)$ \\
Yes & $60(20.7 \%)$ \\
\hline
\end{tabular}

Notes: Data are presented in $\mathrm{n}(\%)$ unless specified otherwise. ${ }^{\text {a }}$ Marital status: married, widow, divorced, or unmarried people were included; beducation level: low (primary school through vocational training), medium (secondary school or vocational training), and high (college or university degree); 'care allowance received by single people making an annual living of $<€ 30.939$ or a combined annual living of $<€ 42.438$; dGOLD stage: I (FEV $\geq 80 \%$ ), II (50\% $\left.\leq \mathrm{FEV}_{1}<80 \%\right)$, III $\left(30 \% \leq \mathrm{FEV}_{1}<50 \%\right)$, IV (FEV $<30 \%$ ); " ${ }^{\circ}$ comorbidities: cardiovascular diseases, respiratory diseases, diabetes, kidney diseases, liver diseases, cancer, connective tissue disorders, paraplegia, osteoporosis and arthrosis, gastrointestinal disorders, anxiety and depression, Parkinson's disease and multiple sclerosis, eczema, dementia, and HIV; 'Current exacerbation: no (no course of antibiotics and/or prednisolone in the past 3 weeks), yes (course of antibiotics and/or prednisolone in the past 3 weeks).

Abbreviations: $\mathrm{BMI}$, body mass index; $\mathrm{FEV}_{1}$, forced expiratory volume in I second; MSPSS, Multidimensional Scale of Perceived Social Support; B-IPQ, Brief Illness Perception Questionnaire; PAM, Patient Activation Measure; SD, standard deviation; HADS, Hospital Anxiety and Depression Scale; GOLD, Global Initiative for Chronic Obstructive Lung Disease; MRC, Medical Research Council; SF-12, Short-Form- 12 Health Survey.

candidate predictors. Pooled estimates of the association, derived from the estimates per imputed dataset as created by SPSS, are used in the "Results" section.

A stepwise backward multiple linear regression analysis was performed in order to identify explanatory variables of activation for self-management. Variables were excluded in order of the highest $P$-value. A significance level of $20 \%$ was used to keep a variable in the model. This method was applied to each of the ten imputation data sets separately and resulted in ten sets of selected variables. The majority method was used to keep variables in the final model, which consisted of variables that were selected in $50 \%$ or more of the ten data sets..$^{38}$ To calculate pooled $R^{2}$ statistics, Fisher's $r$ to $z$ transformation was used..$^{39}$ Assumptions of linearity, multicollinearity $(R>0.8)$, and homoscedasticity were checked and approved. Some continuous variables did not completely meet the assumption for normal distribution. Therefore, generalized linear models were used with robust standard errors in the linear regression analysis.

\section{Results}

In total, 607 eligible COPD patients were invited for this study, of which 315 patients (52\%) agreed to participate. A total of 42 patients were excluded during the process of recruitment and data collection. Finally, 290 participants were found to be eligible for analysis (Figure 1).

The mean age of participants was 67.2 (SD 10.3) and $63.4 \%$ were males. The majority of participants were Dutch (92.4\%), married (66.2\%), unemployed (81.7\%), nonsmokers (68.3\%), and had a low-to-medium education level (81.7\%). Most participants had moderate COPD as mean $\mathrm{FEV}_{1} \%$ predicted was 63.6 (GOLD stage 2 ). In addition, 


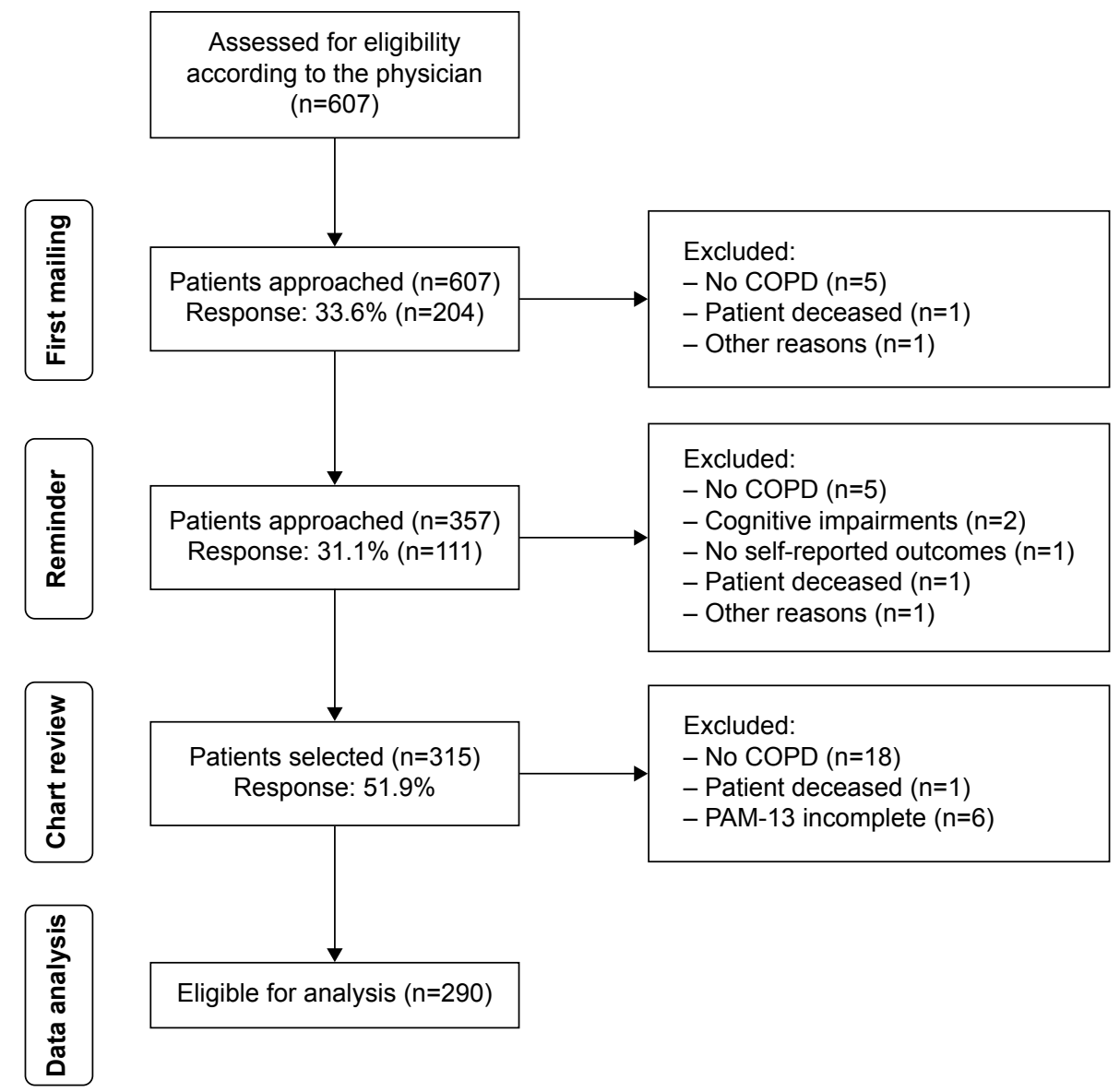

Figure I Flowchart of recruitment.

Abbreviation: PAM, Patient Activation Measure.

a majority of $63.1 \%$ of the participants had a MRC score below three. Nearly half of the population was diagnosed with COPD for more than 5 years $(46.6 \%)$. Other patients' characteristics are detailed in Table 1.

\section{Activation for self-management}

The mean activation score (PAM-13) was 54.7 (SD 10.4). Figure 2 shows the prevalence of different levels of activation for self-management among the study population and details that participants were almost equally distributed in PAM-13 levels 2 and 3 (29.7 vs 33.1, respectively). Poor activation for self-management (level 1) was observed among $22.8 \%$ of the participants. A minority of $14.5 \%$ of the participants was activated for self-management and scored on level 4 (Figure 2).

\section{Determinants associated with activation for self-management}

Univariate associations between determinants and activation for self-management are presented in Table 2. Physical health status, mental health status, anxiety, depression, illness perception, BMI, education level, dyspnea, and GOLD

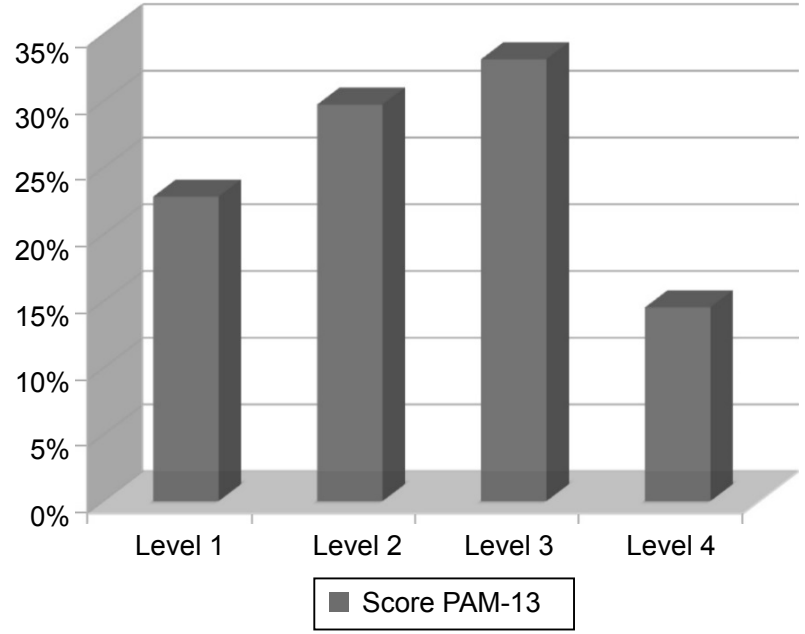

Figure 2 Distribution of different PAM levels.

Notes: Level I ( $\leq 47.0$ ): Disengaged and overwhelmed: Individuals are passive and lack confidence. Knowledge is low, goal-orientation is weak, and adherence is poor. Level 2 (47.I-55. I): Becoming aware, but still struggling: Individuals have some knowledge, but large gaps remain. They believe health is largely out of their control, but can get simple goals. Level 3 (55.2-67.0): Taking action: Individuals have the key facts and are building self-management skills. They strive for the best practice behaviors and are goal-oriented. Level 4 ( $\geq 67.1)$ : Maintaining behaviors and pushing further: Individuals have adopted new behaviors, but may struggle in times of stress or change. Maintaining a healthy lifestyle is a key focus. Description of PAM levels is cited from http://www.insigniahealth.com. ${ }^{23}$

Abbreviation: PAM, Patient Activation Measure. 
Table 2 Univariate linear regression and multiple linear regression to analyze the association between multiple determinants and activation for self-management

\begin{tabular}{|c|c|c|c|c|c|c|}
\hline \multirow{2}{*}{$\begin{array}{l}\text { Association of determinants with } \\
\text { self-management capacity (PAM-13) }\end{array}$} & \multicolumn{3}{|c|}{ Univariate linear regression } & \multicolumn{3}{|c|}{ Multiple linear regression } \\
\hline & $\beta$ & $95 \% \mathrm{Cl}$ & $P$-value & $\beta$ & $95 \% \mathrm{Cl}$ & $P$-value \\
\hline Health status physical component (SF-I2) & 0.12 & $0.08 / 0.17$ & 0.00 & & NA & \\
\hline Health status mental component (SF-12) & 0.11 & $0.06 / 0.16$ & 0.00 & & NA & \\
\hline Anxiety (HADS) & -0.54 & $-0.81 /-0.27$ & 0.00 & -0.35 & $-0.64 /-0.06$ & 0.02 \\
\hline Depression (HADS) & -0.69 & $-0.97 /-0.4 I$ & 0.00 & & NA & \\
\hline IIlness perception (B-IPQ) & -0.26 & $-0.36 /-0.17$ & 0.00 & -0.17 & $-0.28 /-0.06$ & 0.00 \\
\hline Social support (MSPSS) & 0.05 & $-0.01 / 0.12$ & 0.12 & & NA & \\
\hline Age (years) & -0.11 & $-0.22 / 0.01$ & 0.07 & -0.14 & $-0.26 /-0.01$ & 0.03 \\
\hline Sex (female vs male) & 0.25 & $-2.26 / 2.76$ & 0.85 & & NA & \\
\hline BMI $\left(\mathrm{kg} / \mathrm{m}^{2}\right)$ & -0.38 & $-0.64 /-0.13$ & 0.00 & -0.42 & $-0.65 /-0.19$ & 0.00 \\
\hline Ethnicity (other vs Dutch) & -4.14 & $-8.96 / 0.69$ & 0.09 & & NA & \\
\hline Living situation (not alone vs alone) & -1.27 & $-4.05 / 1.50$ & 0.37 & & NA & \\
\hline Education level (low, medium, high) & & & & & NA & \\
\hline Moderate vs low & 2.30 & $-0.32 / 4.92$ & 0.09 & & & \\
\hline High vs low & 4.85 & $1.40 / 8.31$ & 0.01 & & & \\
\hline Financial distress (none, low, high) & & & & & NA & \\
\hline Low vs none & -0.91 & $-3.50 / 1.68$ & 0.49 & & & \\
\hline High vs none & -2.30 & $-6.32 / 1.71$ & 0.26 & & & \\
\hline Care allowance (received vs not received) & 0.23 & $-2.25 / 2.71$ & 0.86 & & NA & \\
\hline Current smoking (never, former, current) & & & & & NA & \\
\hline Former vs never & 3.78 & $-0.56 / 8.13$ & 0.09 & & & \\
\hline Current vs never & 3.98 & $-0.62 / 8.59$ & 0.09 & & & \\
\hline Dyspnea (MRC) (>3 vs $\leq 3)$ & -3.69 & $-6 .|7 /-| .2 \mid$ & 0.00 & & NA & \\
\hline \multicolumn{7}{|l|}{ GOLD stage } \\
\hline GOLD 2 vs I & -3.65 & $-6.37 /-0.93$ & 0.01 & -3.15 & $-5.77 /-0.54$ & 0.02 \\
\hline GOLD 3 vs I & -4.89 & $-8.70 /-1.08$ & 0.01 & -3.37 & $-7.07 / 0.32$ & 0.07 \\
\hline GOLD 4 vs I & -4.02 & $-9.70 / 1.67$ & 0.17 & -2.48 & $-7.62 / 2.67$ & 0.35 \\
\hline Illness duration ( $\leq 2$ years, $2-5$ years, $>5$ years) & & & & & NA & \\
\hline $2-5$ years vs $\leq 2$ years & 1.25 & $-2.60 / 5.09$ & 0.53 & & & \\
\hline$>5$ years vs $\leq 2$ years & -0.44 & $-4.07 / 3.20$ & 0.81 & & & \\
\hline Current exacerbation (no vs yes) & 2.40 & $-0.56 / 5.35$ & 0.11 & & NA & \\
\hline Comorbidity $(\mathrm{CCl})$ & 0.42 & $-0.38 / 1.21$ & 0.30 & 0.79 & $-0.19 / 1.77$ & 0.11 \\
\hline Explained variance of the model $R^{2}=0.172(\mathrm{n}=290)$ & & & & & & \\
\hline
\end{tabular}

Abbreviations: MSPSS, Multidimensional Scale of Perceived Social Support; B-IPQ, Brief Illness Perception Questionnaire; PAM, Patient Activation Measure; HADS, Hospital Anxiety and Depression Scale; GOLD, Global Initiative for Chronic Obstructive Lung Disease; MRC, Medical Research Council; BMI, body mass index; CCl, Charlson comorbidity index; $\mathrm{Cl}$, confidence interval; NA, not applicable; SF-12, Short-Form-12 Health Survey.

stage were significantly associated with activation for selfmanagement $(P<0.05)$.

Multiple linear regression analysis revealed six explanatory determinants of activation for self-management: anxiety, illness perception, BMI, age, GOLD stage, and comorbidities (Table 2).

Increased level of anxiety ( $\beta$ : $-0.35 ; \mathrm{CI}:-0.64$ to -0.06$)$, a more negative illness perception $(-0.17 ;-0.28$ to -0.06$)$, increased BMI $(-0.42 ;-0.65$ to -0.19$)$, increased age $(-0.14 ;-0.26$ to -0.01$)$, increased GOLD stage ( 2 vs $1:-3.15$; -5.77 to -0.54 , 3 vs $1:-3.37 ;-7.07$ to 0.32 ), and less comorbidities $(0.79 ;-0.19$ to 1.77$)$ were associated with a decrease in activation for self-management $(P<0.2)$. For GOLD stage, a statistical significant association was observed specifically in GOLD stage 2 vs $1(P<0.5)$. The explained variance $\left(R^{2}\right)$ of the multivariable model was 0.17 . High correlations were observed between mental and physical health $(R=0.76)$ and anxiety and depression $(R=0.73)$.

\section{Discussion}

This study has provided insight into the prevalence of different levels of activation for self-management and identified patient and disease characteristics associated with activation for self-management in COPD patients. Only a minority of COPD patients was activated for selfmanagement. The main finding was that increased anxiety, 
a more negative illness perception, higher BMI, higher age, more disease severity, and less comorbidities were associated with a lower activation for self-management. These variables explained $17 \%$ of the variance in activation for self-management.

Activation for self-management in the study population, represented by the mean activation score, was lower compared to a previous Dutch study including COPD patients. ${ }^{21}$ This might be explained by the fact that this study focused on various chronic disease patients who were younger (58.7 vs 67.2 years). ${ }^{21}$ In our study, only a minority of participants was activated for self-management (level 4), which indicates major room for improvement. Slightly more than half of the participants were in levels 2 and 3 and nearly a quarter was considered to be a poor self-manager (level 1). In contrast, another Dutch study that focused on activation for self-management in COPD patients showed that most patients were in levels 3 and $4 .{ }^{40}$ Since both other Dutch studies sampled patients from a national panel, this may have positively affected the outcome as these patients might be more activated for self-management.

This study identified anxiety, illness perception, BMI, age, GOLD stage, and comorbidities as explanatory determinants of activation for self-management. This is partly in line with previous studies focusing on self-management in COPD patients. A previous literature review identified anxiety, illness perception, and dyspnea as factors influencing self-management. ${ }^{8}$ In addition, associations with age and disease severity were observed in another study focusing on self-management. ${ }^{18}$ On the contrary, socioeconomic status and social support were expected to be related with self-management, ${ }^{8}$ although no significant association with activation for self-management was observed in our study. This might be due to the large heterogeneity in selfmanagement outcome parameters used in previous studies and our specific focus on activation for self-management. Remarkably, comorbidities and BMI were identified as key determinants in our study, while this has not been reported in previous studies to our knowledge. A previous study using the PAM-13 in COPD patients identified no associations with age and presence of comorbidities, although a small association with dyspnea was found..$^{40}$ In our study, age and comorbidities were identified as key determinants of activation for self-management which may be explained by the fact that we included multiple determinants in our model, investigating the relative influence of each individual determinant, and the fact that patients in our study had relatively more comorbidities.
The identified determinants were partly in line with findings from our larger study focusing on determinants for selfmanagement in various chronic diseases. ${ }^{16}$ In line with that study, the age, BMI and illness perception were identified as key determinants. On the contrary, education level, financial distress, physical health status, depression, and social support were not identified as key determinants in this current study. In this study, anxiety was identified as a key determinant. It is important to note that anxiety was highly correlated with depression and mental health status. This may indicate that emotional distress in general is an important determinant of self-management behavior. Furthermore, contrasting was that disease severity emerged as a key determinant in this study, though not in the larger study. This might be explained by the fact that disease severity was standardized for various chronic diseases in the larger study, leading to broader categories of severity. Finally, comorbidity was a key determinant in this study, indicating that COPD patients with several comorbidities seem more activated for self-management. This might be due to the fact that these patients already have more experience with health care and know how to cope with their disease. The results of this study indicate that anxiety, disease severity, and comorbidity were more important in identifying the level of activation in COPD patients than they were in the mixed group of patients with various chronic conditions.

The explained variance was $17 \%$, which is lower compared to previous studies on explanatory variables of self-management in COPD patients (varying from $31 \%$ to $34 \%$ ). ${ }^{18,41}$ The identified key determinants could only explain the variance of activation for self-management to a limited extent. The remaining variance may be explained by other types of factors influencing activation for self-management, for example, self-efficacy or received self-management support from health care professionals. Self-efficacy was not included in this study since the PAM-13 already includes items focusing on self-efficacy. However, in social cognitive theory, selfefficacy is considered to be an important intermediate in the causal chain toward adoption of self-management skills and behavioral change. ${ }^{42,43}$

An important strength of this study was that a wide range of determinants was analyzed simultaneously in a relatively large study population. Inclusion from both primary and secondary care had a positive impact on the generalizability of the results since this maximizes variation in COPD severity. Finally, the response rate of more than $50 \%$ was higher than the expected rate of $40 \%$, which strengthens the external validity of this study. A limitation of this study was that patients were recruited by physicians in different settings, which may have resulted in 
selection bias. In primary care, a few patients were considered eligible by their physician based on GOLD stage, while lung function data were missing. Those participants were included in the analysis when GOLD stage was explicitly listed in the chart and patients received active treatment for their COPD. Furthermore, in this study less non-native patients were included than expected based on the data of the Dutch population, ${ }^{44}$ which might have been due to language barriers.

The acquired knowledge on explanatory determinants of activation for self-management is important for all health care professionals supporting COPD patients in self-management, as it allows them to make a risk assessment of inadequate engagement in self-management based on an individual patient profile. Based on the study results, specific attention should be paid to relatively older patients, with a relatively high weight, a more negative illness perception, more severe COPD, less comorbidities, and emotional disturbances. This stresses the need for adequate assessment on patient-related factors that can be influenced such as illness perception, anxiety, and BMI, as improving these factors may lead to increased quality of life or health status. ${ }^{45,46}$ For example, health care professionals should pay more attention to identifying negative illness perceptions by asking patients how they experience their COPD and how COPD symptoms influence their daily lives, ${ }^{46}$ so that they can anticipate on these perceptions in future consultations.

For patients at risk for inadequate engagement in selfmanagement, intensifying self-management support seems important to increase the likelihood of engagement in selfmanagement. First, adequate assessment by health care professionals on patient knowledge, skills, and confidence is needed to identify problem areas allowing them to anticipate on these individual problem areas with tailored strategies. Intensifying self-management support may then consist of spending more time on education or to provide additional materials to increase patients' knowledge, to amplify action planning and decision support to increase patients skills, or to add reinforcement consultations to increase patients confidence for self-management.

The knowledge on determinants of activation for selfmanagement may help health care professionals to make a first step in targeting and tailoring their interventions. Assessment on engagement in self-management based on patient profiles, and identifying behavioral needs, may contribute to individualizing self-management interventions. Dose, content, and modus of self-management interventions should then be tailored to individual patient needs and capabilities.
More research is needed to investigate barriers and facilitators of activation for self-management in COPD patients including a focus not only on other patient-related factors, such as self-efficacy, but also on provider and health care system characteristics. These studies should focus on identifying causal relationships between determinants and activation for self-management. Longitudinal studies are required to determine key determinants of change in activation for self-management. This knowledge is essential to eliminate barriers of activation for self-management and will contribute to targeting and tailoring of self-management interventions.

\section{Conclusion}

This study showed that only a minority of COPD patients is activated for self-management, which implies that there is great potential for improvement in self-management and subsequently in health outcomes. This study found that increased anxiety, a more negative illness perception, increased BMI, increased age, increased disease severity, and less comorbidities were associated with a decrease in activation for selfmanagement in COPD patients. This knowledge contributes to identification of patients at risk of inadequate engagement in self-management activities, which is an essential first step toward targeting and tailoring individualized self-management interventions in the future. To be able to thoroughly understand the complex causal mechanisms toward change in self-management behavior, future research is needed.

\section{Acknowledgment}

We thank all the patients who participated and acknowledge all health centers for their cooperation in this study.

\section{Disclosure}

The authors report no conflict of interest in this work.

\section{References}

1. Global Initiative for Chronic Obstructive Lung Disease (GOLD). Global strategy for the diagnosis, management, and prevention of chronic obstructive pulmonary disease. Updated 2016. Available from: http:// www.goldcopd.org/uploads/users/files/GOLD_Report\%202016.pdf. Accessed February 2, 2016.

2. World Health Organization. The global burden of disease. Updated 2004. Available from: http://www.who.int/healthinfo/global_burden_disease/ GBD_report_2004update_full.pdf?ua=1. Accessed October 28, 2015.

3. Mathers CD, Loncar D. Projections of global mortality and burden of disease from 2002 to 2030. PLoS Med. 2006;3(11):e442.

4. Bourbeau J, van der Palen J. Promoting effective self-management programmes to improve COPD. Eur Respir J. 2009;33(3):461-463.

5. Zwerink M, Brusse-Keizer M, van der Valk PD, et al. Self management for patients with chronic obstructive pulmonary disease. Cochrane Database Syst Rev. 2014;3:CD002990. 
6. SpruitMA, Singh SJ, Garvey C, etal. An official American Thoracic Society/ European Respiratory Society statement: key concepts and advances in pulmonary rehabilitation. Am J Respir Crit Care Med. 2013;188(8): e13-e64

7. Barlow J, Wright C, Sheasby J, Turner A, Hainsworth J. Self-management approaches for people with chronic conditions: a review. Patient Educ Couns. 2002;48(2):177-187.

8. Disler RT, Gallagher RD, Davidson PM. Factors influencing selfmanagement in chronic obstructive pulmonary disease: an integrative review. Int J Nurs Stud. 2012;49(2):230-242.

9. Bourbeau J, Nault D. Self-management strategies in chronic obstructive pulmonary disease. Clin Chest Med. 2007;28(3):617-628, vii.

10. Effing TW, Bourbeau J, Vercoulen J, et al. Self-management programmes for COPD: moving forward. Chron Respir Dis. 2012;9(1):27-35.

11. Adams SG, Smith PK, Allan PF, Anzueto A, Pugh JA, Cornell JE. Systematic review of the chronic care model in chronic obstructive pulmonary disease prevention and management. Arch Intern Med. 2007; 167(6):551-561

12. Bourbeau J, Julien M, Maltais F, et al. Reduction of hospital utilization in patients with chronic obstructive pulmonary disease: a disease-specific self-management intervention. Arch Intern Med. 2003;163(5):585-591.

13. Trappenburg J, Jonkman N, Jaarsma T, et al. Self-management: one size does not fit all. Patient Educ Couns. 2013;92(1):134-137.

14. Hibbard JH, Mahoney ER, Stockard J, Tusler M. Development and testing of a short form of the patient activation measure. Health Serv Res. 2005;40(6 Pt 1):1918-1930.

15. Hibbard JH, Mahoney ER, Stock R, Tusler M. Do increases in patient activation result in improved self-management behaviors? Health Serv Res. 2007;42(4):1443-1463.

16. Bos-Touwen I, Schuurmans M, Monninkhof EM, et al. Patient and disease characteristics associated with activation for self-management in patients with diabetes, chronic obstructive pulmonary disease, chronic heart failure and chronic renal disease: a cross-sectional survey study. PLoS One. 2015;10(5):e0126400.

17. Cramm JM, Nieboer AP. Self-management abilities, physical health and depressive symptoms among patients with cardiovascular diseases, chronic obstructive pulmonary disease, and diabetes. Patient Educ Couns. 2012;87(3):411-415.

18. Warwick M, Gallagher R, Chenoweth L, Stein-Parbury J. Self-management and symptom monitoring among older adults with chronic obstructive pulmonary disease. J Adv Nurs. 2010;66(4):784-793.

19. Peduzzi P, Concato J, Kemper E, Holford TR, Feinstein AR. A simulation study of the number of events per variable in logistic regression analysis. J Clin Epidemiol. 1996;49(12):1373-1379.

20. Hibbard JH, Stockard J, Mahoney ER, Tusler M. Development of the Patient Activation Measure (PAM): conceptualizing and measuring activation in patients and consumers. Health Serv Res. 2004;39(4 Pt 1): 1005-1026.

21. Rademakers J, Nijman J, van der Hoek L, Heijmans M, Rijken M. Measuring patient activation in the Netherlands: translation and validation of the American short form Patient Activation Measure (PAM13). BMC Public Health. 2012;12(1):577.

22. Insignia Health. Patient Activation Measure (PAM) 13, licence materials. 2011.

23. Insignia Health. Patient Activation Measure (PAM) Survey levels. Available from: http://www.insigniahealth.com/products/pam-survey. Accessed March 10, 2016.

24. Ware J Jr, Kosinski M, Keller SD. A 12-Item Short-Form Health Survey: construction of scales and preliminary tests of reliability and validity. Med Care. 1996;34(3):220-233.

25. Aaronson NK, Muller M, Cohen PD, et al. Translation, validation, and norming of the Dutch language version of the SF-36 Health Survey in community and chronic disease populations. J Clin Epidemiol. 1998;51(11):1055-1068.

26. Mols F, Pelle AJ, Kupper N. Normative data of the SF-12 health survey with validation using postmyocardial infarction patients in the Dutch population. Qual Life Res. 2009;18(4):403-414.
27. Zigmond AS, Snaith RP. The hospital anxiety and depression scale. Acta Psychiatr Scand. 1983;67(6):361-370.

28. Spinhoven P, Ormel J, Sloekers PP, Kempen GI, Speckens AE, Van Hemert AM. A validation study of the Hospital Anxiety and Depression Scale (HADS) in different groups of Dutch subjects. Psychol Med. 1997;27(2):363-370.

29. De Raaij EJ, Schroder C, Maissan FJ, Pool JJ, Wittink H. Crosscultural adaptation and measurement properties of the Brief Illness Perception Questionnaire-Dutch Language Version. Man Ther. 2012; 17(4):330-335.

30. Zimet GD, Dahlem NW, Zimet SG, Farley GK. The Multidimensional Scale of Perceived Social Support. J Pers Assess. 1988(52):30-41.

31. Zimet GD, Powell SS, Farley GK, Werkman S, Berkoff KA. Psychometric characteristics of the Multidimensional Scale of Perceived Social Support. J Pers Assess. 1990;55(3-4):610-617.

32. Pedersen SS, Spinder H, Erdman RA, Denollet J. Poor perceived social support in implantable cardioverter defibrillator (ICD) patients and their partners: cross-validation of the multidimensional scale of perceived social support. Psychosomatics. 2009;50(5):461-467.

33. Bestall JC, Paul EA, Garrod R, Garnham R, Jones PW, Wedzicha JA. Usefulness of the Medical Research Council (MRC) dyspnoea scale as a measure of disability in patients with chronic obstructive pulmonary disease. Thorax. 1999;54(7):581-586.

34. Charlson ME, Pompei P, Ales KL, MacKenzie CR. A new method of classifying prognostic comorbidity in longitudinal studies: development and validation. J Chronic Dis. 1987;40(5):373-383.

35. Sundararajan V, Henderson T, Perry C, Muggivan A, Quan H, Ghali WA. New ICD-10 version of the Charlson comorbidity index predicted inhospital mortality. J Clin Epidemiol. 2004;57(12):1288-1294.

36. IBM Corp. IBM SPSS Statistics for Windows, Version 21.0. Armonk, NY: IBM Corp.; 2012.

37. Donders AR, van der Heijden GJ, Stijnen T, Moons KG. Review: a gentle introduction to imputation of missing values. J Clin Epidemiol. 2006;59(10):1087-1091

38. Vergouwe Y, Royston P, Moons KG, Altman DG. Development and validation of a prediction model with missing predictor data: a practical approach. J Clin Epidemiol. 2010;63(2):205-214.

39. Harel $\mathrm{O}$. The estimation of R2 and adjusted R2 in incomplete data sets using multiple imputation. Journal of Applied Statistics. 2009; 36(10):1109-1118.

40. Baan D, Heijmans M, Spreeuwenberg P, Rijken M. Zelfmanagement vanuit het perspectief van mensen met astma of COPD. Available from: http://www.nivel.nl/sites/default/files/bestanden/Rapport-Zelfmanagement-mensen-met-COPD-of-Astma.pdf. Accessed March 21, 2016

41. Wang KY, Sung PY, Yang ST, Chiang CH, Perng WC. Influence of family caregiver caring behavior on COPD patients' self-care behavior in Taiwan. Respir Care. 2012;57(2):263-272.

42. Bourbeau J. Self-management interventions to improve outcomes in patients suffering from COPD. Expert Rev Pharmacoecon Outcomes Res. 2004;4(1):71-77.

43. Bandura A. Self-efficacy: toward a unifying theory of behavioral change. Psychol Rev. 1977;84(2):191-215.

44. Sanderse C, Verweij A, Beer J de. Etniciteit: Wat is de huidige situatie? Available from: http://www.nationaalkompas.nl/bevolking/etniciteit/ huidig/. Accessed March 1, 2016.

45. Tsiligianni I, Kocks J, Tzanakis N, Siafakas N, van der Molen T. Factors that influence disease-specific quality of life or health status in patients with COPD: a review and meta-analysis of Pearson correlations. Prim Care Respir J. 2011;20(3):257-268.

46. Weldam SW, Lammers JW, Heijmans MJ, Schuurmans MJ. Perceived quality of life in chronic obstructive pulmonary disease patients: a crosssectional study in primary care on the role of illness perceptions. BMC Fam Pract. 2014;15:140. 


\section{Publish your work in this journal}

The International Journal of COPD is an international, peer-reviewed journal of therapeutics and pharmacology focusing on concise rapid reporting of clinical studies and reviews in COPD. Special focus is given to the pathophysiological processes underlying the disease, intervention programs, patient focused education, and self management protocols.

This journal is indexed on PubMed Central, MedLine and CAS. The manuscript management system is completely online and includes a very quick and fair peer-review system, which is all easy to use. Visit http://www.dovepress.com/testimonials.php to read real quotes from published authors. 